ANDRZEJ RACZYŃSKI (Wrocław)

\title{
A MODEL OF EVOLUTION OF TEMPERATURE AND DENSITY OF IONS IN AN ELECTROLYTE
}

Abstract. We study existence and nonexistence of solutions (both stationary and evolution) for a parabolic-elliptic system describing the electrodiffusion of ions. In this model the evolution of temperature is also taken into account. For stationary states the existence of an external potential is also assumed.

1. Introduction. In this paper we are interested in the temporal evolution of the spatial density of ions in an electrolyte confined to a container $\Omega \subset \mathbb{R}^{n}, n=2,3$. We consider a simplified, idealized situation when the electrolyte contains only cations, i.e. positively charged particles. The reasoning in the more realistic case when several species $u_{1}, \ldots, u_{k}$ of charged particles are considered is similar but the notations would be a bit more complicated (cf. [7], [15], [16]).

The model we consider consists of three equations

$$
\begin{aligned}
& u_{t}=\nabla \cdot\left(\nabla u+\frac{u}{\theta} \nabla \varphi\right), \\
& \Delta \varphi=-u, \\
& E=q_{0} \theta+\frac{1}{2} \int_{\Omega} u \varphi d x .
\end{aligned}
$$

The first one, a nonlinear parabolic equation of drift-diffusion type, describes the evolution of the density $u=u(x, t)$ of the ions in the field generated by the electric potential $\varphi=\varphi(x, t)$, produced by the ions through collective effects, and coupled with the density via the Poisson equation (2). These equations are supplemented with the energy relation (3) which determines

2000 Mathematics Subject Classification: 35Q, 35K60, 35K57, 35B40, 82C21.

Key words and phrases: mean field equations, drift-diffusion and energy-transport systems, nonlinear parabolic-elliptic system, electrodiffusion of ions.

The preparation of this paper was supported by the KBN grant 2/P03A/011/19. 
the temperature $\theta=\theta(t)$ uniform in the domain $\Omega \subset \mathbb{R}^{n}$. The constant $q_{0}=\int_{\Omega} u$ denotes the total charge of the ions.

The system can be considered as a version of the so-called ChavanisSommeria-Robert model, with the electrical interaction replacing the gravitational one in [11].

The motivation to study such a model lies in its location midway between isothermal models of mean field type for electrolytes and semiconductors going back to Nernst, Planck, Debye and Hückel ([1], [4], [7], [13]) and general energy-transport models of Streater type which include a nonlinear heat equation for evolution of temperature ([3], [2], [5], [10], [17], [18]).

Although these three models are strongly related, there are also important differences between them. The isothermal model has a long history and is well studied. The model of Streater type (with $\theta=\theta(x, t)$ ), which is relatively new, is much more complicated and causes many difficulties. In particular, there are (to the best of my knowledge) no results on local existence of solutions for the general Streater model with interaction ([2], [3]). The model with temperature depending only on time is much simpler than the general one, but retains its mathematical properties and on the other hand is more complicated than the isothermal model. However, the model with temperature depending on time and space is usually more realistic, but because of mathematical difficulties we have restricted ourselves only to time dependence.

On the other hand, the model can be treated as an extension of those studied in [7], [13], [14] but with nonconstant temperature.

The next problem we will consider is the existence of stationary solutions for the model (1)-(3) in the presence of an external potential. Thus, the model reads

$$
\begin{aligned}
& u_{t}=\nabla \cdot\left(\nabla u+\frac{u}{\theta} \nabla(\varphi+V)\right), \\
& \Delta \varphi=-u, \\
& E=q_{0} \theta+\frac{1}{2} \int_{\Omega} u \varphi+\int_{\Omega} u V,
\end{aligned}
$$

where $V: \mathbb{R}^{n} \rightarrow \mathbb{R}$ denotes a given external potential.

For more physical details we refer the reader to [7], [9], [15].

In this paper we assume that the external potential $V$ is singular, of the form $V(x)=-q^{*} E_{n}(x)$, where $q^{*} \neq 0$ and $E_{n}$ is the fundamental solution of the Laplacian $\left(E_{2}(z)=(2 \pi)^{-1} \log |z|, E_{n}(z)=-\left((n-2) \sigma_{n}\right)^{-1}|z|^{2-n}\right.$ for $n \geq 3$, where $\sigma_{n}$ is the area of the unit sphere in $\left.\mathbb{R}^{n}\right)$. This means that $V$ is produced by an additional point charge $q^{*}$ fixed at the origin. Such a singular potential may lead to difficult questions considered in [15, Section 2.4], [16, Chapter 2, Section 2] and [7]. 
The problem (4)-(6) with $q^{*}<0$ describes the situation when a charge $q^{*}$ fixed at the origin is surrounded by ions of opposite sign moving in a solute. The case $q^{*}>0$ means that the ions and the charge are of the same sign.

The system (1)-(3) is supplemented with the initial condition

$$
u(x, 0)=u_{0}(x)
$$

and with the boundary conditions

$$
\begin{aligned}
\frac{\partial u}{\partial \nu}+\frac{u}{\theta} \frac{\partial \varphi}{\partial \nu} & =0 & & \text { on } \partial \Omega, \\
\varphi & =0 & & \text { on } \partial \Omega,
\end{aligned}
$$

where $\nu$ denotes the exterior unit normal to $\partial \Omega$. Those conditions are a mathematical simplification of the situation when the total charge of ions in $\Omega$ is preserved (because of the no-flux condition (8) across the boundary), i.e. $\int_{\Omega} u(x, t) d x=\int_{\Omega} u_{0}(x) d x=: q_{0}$. The condition (9) means that the boundary $\partial \Omega$ is grounded. Let us notice that the condition $\varphi=$ const on the boundary is equivalent to (9) since the equations (4)-(5) are invariant under the shift $\varphi \mapsto \varphi+$ const (only in (6) the additional term $\frac{1}{2} q_{0}$ const should be added). This means that in the radially symmetric case all Dirichlet conditions are equivalent to (9).

A given value of the energy $E$ determines the initial temperature $\theta_{0}$ due to the relation (3) (the potential $\varphi_{0}$ is obtained from $u_{0}$ via (2)).

Similarly, the system (4)-(6) is supplemented with the initial condition (7), boundary condition (9) and

$$
\frac{\partial u}{\partial \nu}+\frac{u}{\theta} \frac{\partial}{\partial \nu}(\varphi+V)=0 \quad \text { on } \partial \Omega
$$

It was proved in [1], [2] and [4] that a local-in-time solution to (1)(2), (7)-(9) with constant temperature $\theta \equiv 1$ exists. Moreover, under some assumptions imposed on the dimension $n$ or the initial data $u_{0}$, this solution is global and tends to a unique steady state as $t$ goes to $\infty$.

It is worth noting that the existence of solutions of the isothermal problem with $q^{*}<0$ depends on $n$ and $q^{*}([7])$. For instance, in the radially symmetric case, if $n=2$ and $q^{*}>-4 \pi$ the solution is global, whereas for $q^{*}<-4 \pi$ or $n \geq 3$ and $q^{*}<0$ there are no local-in-time solutions. In case $q^{*} \geq 0$, the global solution exists for each $q^{*} \geq 0$ and tends asymptotically, as time goes to infinity, to the unique steady state solution ([13]).

We are interested in the existence and uniqueness of stationary solutions to the problem (4)-(7), (9), (10). In this paper we prove the existence of radially symmetric solutions for all values $q^{*}, q_{0}>0$ and $E>E_{0}($ for $n=2$ ). For $n=3$ we get an analogous conclusion for $q^{*}>0$ and nonexistence of solutions for $q^{*}<0$. 
Considering the evolution problem (with $V=0$ ) we will prove the existence of local weak solutions of the problem (1)-(3), (7)-(9). Next we will prove that the solutions obtained can be continued in time for all $t \geq 0$ (for $n=2)$.

Energy. In our models we assume that the energy is the sum of the potential energy $\int_{\Omega}\left(\frac{1}{2} u \varphi+u V\right)$ and the one connected with the thermal motion of the particles (also connected with the thermal energy), $\int_{\Omega} u \theta$. By assumption (6) the energy is constant.

Since we consider the idealized situation and take only ions of one sign into account we do not take the thermal capacity of the electrolyte into consideration. In a more realistic situation of a classical electrolyte (with ions of opposite signs) the isothermal case would be a better approximation of a real situation since the thermal capacity of the electrolyte is much larger then the ions' contribution to the thermal energy.

Entropy. For sufficiently smooth solutions the function $W(t)$ defined as

$$
W=W(t)=\int u(\log u-\log \theta)
$$

plays the role of (neg)entropy.

Indeed, from the energy relation (6) we have $q_{0} \theta_{t}=-\frac{1}{2} \int_{\Omega}(u \varphi)_{t}+\int_{\Omega} V u_{t}$. Due to (4) and (5) the sum of these integrals is equal to $\int_{\Omega} \nabla(\varphi+V) \cdot(\nabla u+$ $\left.\frac{u}{\theta} \nabla(\varphi+V)\right)$.

Thus, we arrive at

$$
\frac{d W}{d t}=-\int_{\Omega} u^{-1}\left(\nabla u+\frac{u}{\theta} \nabla(\varphi+V)\right)^{2},
$$

so $d W / d t \leq 0$, which implies that $W$ is a decreasing function of time.

Notation. $|f|_{p}$ (resp. $\|f\|_{k}$ ) is the $L^{p}(\Omega)$ (resp. $H^{k}(\Omega)$ ) norm of the function $f$. The constant $C$ denotes various inessential constants and may vary from line to line.

2. Evolution problem without external potential. In this section we prove the existence of solutions of the problem (1)-(3), (7)-(9).

We begin with an a priori lower bound estimate for the temperature valid in any bounded $n$-dimensional domain.

LEMma 2.1. Each solution $\langle u, \theta, \varphi\rangle$ of the problem (1)-(3), (7)-(9) with $\theta_{0}>0$ has its temperature bounded from below by a constant depending on the initial data,

$$
\theta(t) \geq \theta_{1}=\theta_{1}\left(u_{0}, \theta_{0}, \Omega\right)>0
$$

for each $t \geq 0$ for which the solution exists. 
Proof. The proof is based on the fact that the entropy $W$ is a nonincreasing function. Indeed, for $W(t)=\int_{\Omega} u(\log u-\log \theta)$ we have

$$
\begin{aligned}
q_{0} \log \theta & =\int_{\Omega} u \log \theta \geq \int_{\Omega} u \log u-W(0) \\
& \geq \max \left\{-e^{-1}|\Omega|, q_{0} \log \frac{q_{0}}{|\Omega|}\right\}-W(0) \geq-e^{-1}|\Omega|-W(0) .
\end{aligned}
$$

The second inequality is a consequence of the Jensen inequality applied to the convex function $s \mapsto s \log s$, and the last one follows from the fact that $\min _{s \in \mathbb{R}^{+}} s \log s=-e^{-1}$. Therefore, the lower bound in Lemma 2.1 is satisfied with a temperature $\theta_{1}>0$.

The next lemma gives an a priori upper bound for the temperature. Let us notice that this is valid in all dimensions and is not a specifically two-dimensional result as in the gravitational case (see [8]).

Lemma 2.2. Let $\langle u, \theta, \varphi\rangle$ be a solution of the problem (1)-(3), (7)-(9). Then

$$
\theta(t) \leq \theta_{2}=\theta_{2}\left(u_{0}, \theta_{0}, \Omega\right)<\infty
$$

for each $t \geq 0$ for which the solution exists.

Proof. We have

$$
q_{0} \theta-E=-\frac{1}{2} \int_{\Omega} u \varphi=-\frac{1}{2} \int_{\Omega}|\nabla \varphi|^{2} \leq 0,
$$

so

$$
\theta \leq \frac{E}{q_{0}}=: \theta_{2}
$$

2.1. Local existence of solutions. Before we prove the existence of solutions for (1)-(3), (7)-(9), let us recall the definition of weak solutions.

We call function $u \in L^{\infty}\left((0, T) ; L^{2}(\Omega)\right) \cap L^{2}\left((0, T) ; H^{1}(\Omega)\right)$ a weak solution of the problem (1)-(3), (7)-(9) if it satisfies the equations below for $v=u$, and for each test function $\eta \in H^{1}(\Omega \times(0, T))$ and for a.e. $t \in(0, T)$,

$$
\begin{gathered}
\int_{\Omega} u(x, t) \eta(x, t) d x-\int_{0}^{t} \int_{\Omega} u \eta_{t} d x d \tau+\int_{0}^{t} \int_{\Omega}\left(\nabla u+\frac{u}{\theta} \nabla \varphi\right) \cdot \nabla \eta d x d \tau \\
=\int_{\Omega} u_{0}(x) \eta(x, 0) d x \\
\Delta \varphi=-v \\
E=q_{0} \theta+\frac{1}{2} \int_{\Omega} v \varphi d x
\end{gathered}
$$


The temperature $\theta$ determined from the formula (15), i.e.

$$
\theta(u)(t)=q_{0}^{-1}\left(E-\frac{1}{2} \int_{\Omega} u(x, t) \varphi(x, t) d x\right),
$$

should be a continuous function.

For a given $T_{0}>0$ define the space $\mathcal{X}=L^{4}\left(\left(0, T_{0}\right) ; L^{2}(\Omega)\right)$ and the operator $\mathcal{T}: \mathcal{X} \rightarrow \mathcal{X}$ such that $u:=\mathcal{T}(v)$ is a solution of $(13)-(15)$ with the given function $v$. Let $B=B_{R}(0)$ be the ball of radius $R>0$ in $\mathcal{X}$.

The assumption $u_{0} \in L^{p}(\Omega)$, with $p>1$, implies local existence of solutions.

THEOREM 2.3. The problem (13)-(15) considered in bounded domains $\Omega \subset \mathbb{R}^{n}(n=2,3)$ with $0 \leq u_{0} \in L^{p}(\Omega), p>n / 2$ and $\theta_{0}>0$ has a unique local solution $u$. This solution belongs to $L_{\mathrm{loc}}^{\infty}\left((0, T) ; L^{p}(\Omega)\right)$ for some $T>0$, and satisfies $u \geq 0, \theta>0$.

Proof. Let us begin with the case $p=2$; the general case of $p>n / 2$ can be treated by a standard approximation procedure as in [4, Th. 2(ii)].

We will prove that any weak solution $u$ satisfies $u_{t} \in L^{2}\left((0, T) ;\left(H^{1}(\Omega)\right)^{\prime}\right)$, so $\theta$ determined from (15), i.e. $\theta(u)(t)=q_{0}^{-1}\left(E-\frac{1}{2} \int_{\Omega} u(x, t) \varphi(x, t) d x\right)$, is a continuous function on $[0, T]$.

The Sobolev inequality for the functions $\varphi$ and $v$ connected by (14), i.e.

$$
|\nabla \varphi|_{q} \leq C|v|_{p} \quad \text { for } 1>\frac{1}{q}=(\geq) \frac{1}{p}-\frac{1}{n}, n>p>\frac{n}{2},
$$

taken for $n=2, q=4 / 3, p=4 / 5$ (resp. $n=3, q=4, p=12 / 7$ ) and the additional assumption $\theta(v) \geq \theta_{0} / 2>0$ for $t \leq T_{0}$ allow us to obtain the inequality

$$
\left|\frac{\nabla \varphi}{\theta}\right|_{1} \leq \frac{C}{\theta_{0}}\left(|v|_{2}+1\right) .
$$

On the other hand, the Calderón-Zygmund inequality for bounded domains ([12, Chapter 9.4]) implies ([6])

$$
\left|\nabla\left(\frac{\nabla \varphi}{\theta}\right)\right|_{2} \leq \frac{C}{\theta_{0}}\left(|v|_{2}+1\right) .
$$

Inequality (16) allows us to get $|\nabla \varphi|_{6} \leq C\left(|v|_{2}+1\right.$ ) (for $n=2$, we take $q=6, p=3 / 2$; for $n=3, q=6, p=2$ ).

These inequalities, as in [6, Th. 1], guarantee the existence of a unique solution $u=\mathcal{T}(v)$ to the problem $(13)-(15)$ on some interval $\left[0, T_{1}\right]\left(T_{1}=\right.$ $\left.T_{1}\left(u_{0}, \Omega\right)\right)$.

Now we prove that $u_{t} \in L^{2}\left((0, T) ;\left(H^{1}(\Omega)\right)^{\prime}\right)$. The interpolation inequality

$$
|u|_{3} \leq C^{1 / 2}\|u\|_{1}^{1 / 2}|u|_{2}^{1 / 2} \quad \text { for } u \in H^{1} \cap L^{2}(n=2,3),
$$


and the energy inequality for the problem (13)-(15), i.e.

$$
\begin{aligned}
\frac{1}{2} \frac{d}{d t}|u|_{2}^{2}+|\nabla u|_{2}^{2} & \leq \int_{\Omega}\left|\frac{u}{\theta} \nabla u \cdot \nabla \varphi\right| d x \leq C\left|\frac{u}{\theta_{0}}\right|_{3}|\nabla u|_{2}|\nabla \varphi|_{6} \\
& \leq \frac{C}{\theta_{0}}|\nabla u|_{2}\|u\|_{1}^{1 / 2}|u|_{2}^{1 / 2}\left(|v|_{2}+1\right) \\
& \leq \frac{C}{\theta_{0}}\|u\|_{H^{1}(\Omega)}^{3 / 2}|u|_{2}^{1 / 2}\left(|v|_{2}+1\right) \\
& \leq \frac{1}{2}\|u\|_{1}^{2}+\frac{C}{\theta_{0}^{4}}|u|_{2}^{2}\left(|v|_{2}+1\right)^{4}
\end{aligned}
$$

imply that

$$
\frac{1}{2} \frac{d}{d t}|u|_{2}^{2}+\frac{1}{2}|\nabla u|_{2}^{2} \leq \frac{C}{\theta_{0}^{4}}|u|_{2}^{2}\left(|v|_{2}+1\right)^{4}
$$

Integrating the above inequality over $[0, t]$ and using the Gronwall lemma we get, for $v \in B_{R} \subset \mathcal{X}$,

$$
\begin{aligned}
|u(t)|_{2}^{2}+\int_{0}^{t}|\nabla u|_{2}^{2} d \tau & \leq\left|u_{0}\right|_{2}^{2} \exp \left(\frac{C}{\theta_{0}^{4}}\left(\|v\|_{L^{4}\left((0, T) ; L^{2}(\Omega)\right)}^{4}+1\right)\right) \\
& \leq\left|u_{0}\right|_{2}^{2} \exp \left(C(R+1) / \theta_{0}^{4}\right) .
\end{aligned}
$$

This implies that

$$
\begin{aligned}
\|u\|_{L^{4}\left((0, t) ; L^{2}(\Omega)\right)}^{4} & \leq A t \quad \text { for } t \in\left[0, T_{1}\right], \\
\|\nabla u\|_{L^{2}\left(\left(0, T_{1}\right) ; L^{2}(\Omega)\right)}^{2} & \leq B .
\end{aligned}
$$

Now we estimate the required norm for $u_{t}$. For any function $\chi \in H^{1}(\Omega)$ we have

$$
\begin{aligned}
\left|\int_{\Omega} u_{t} \chi d x\right| & \leq\left|\int_{\Omega} \nabla u \cdot \nabla \chi d x\right|+\left|\int_{\Omega} \frac{u}{\theta} \nabla \varphi \cdot \nabla \chi\right| \\
& \leq\|u\|_{1}\|\chi\|_{1}+\frac{C}{\theta_{0}}|u|_{3}|\nabla \varphi|_{6}\|\chi\|_{1} \\
& \leq\|u\|_{1}\|\chi\|_{1}+\frac{C}{\theta_{0}}\|u\|_{1}^{1 / 2}|u|^{1 / 2}\left(|v|_{2}+1\right)\|\chi\|_{1} .
\end{aligned}
$$

Thus

$$
\|u\|_{\left(H^{1}(\Omega)\right)^{\prime}} \leq\|u\|_{1}+\frac{C}{\theta_{0}}\|u\|_{1}^{1 / 2}|u|_{2}^{1 / 2}\left(|v|_{2}+1\right)
$$

and

$$
\begin{aligned}
\left\|u_{t}\right\|_{L^{2}\left(\left(0, T_{1}\right) ;\left(H^{1}(\Omega)\right)^{\prime}\right)}^{2} & \leq \int_{0}^{T_{1}}\left(\|u\|_{1}+\frac{C}{\theta_{0}}\|u\|_{1}^{1 / 2}|u|_{2}^{1 / 2}\left(|v|_{2}+1\right)\right)^{2} \\
& \leq C\left(A, B, T_{1}, \Omega, R\right)
\end{aligned}
$$




$$
\left\|u_{t}\right\|_{L^{2}\left(\left(0, T_{1}\right) ;\left(H^{1}(\Omega)\right)^{\prime}\right)} \leq D .
$$

Let $\mathcal{W}$ be the subset of $B \subset L^{4}\left(\left(0, T_{2}\right) ; L^{2}(\Omega)\right), T_{2} \leq T_{1}$, of $v$ 's which satisfy $v(x, 0)=u_{0}$, and the estimates above ((17) and (18)) for $v$ instead of $u$. These conditions guarantee the compactness of $\mathcal{W}$ in $\mathcal{X}$.

Now we need to guarantee that the condition for the temperature, i.e. $\theta(u)>\theta_{0} / 2$, is satisfied.

Indeed, we have

$$
\left|\frac{d}{d t} \theta(v)(t)\right|=\left|\int_{\Omega} v_{t} \varphi\right| \leq\left\|u_{t}\right\|_{\left(H^{1}(\Omega)\right)^{\prime}}\|\varphi\|_{1} \leq C\left\|u_{t}\right\|_{\left(H^{1}(\Omega)\right)^{\prime}}|u|_{2} .
$$

The last inequality is a consequence of (16) for $n=2, q=2, p=3 / 2$ (resp. $n=3, q=4, p=12 / 7)$. We have

$$
\theta(v)(t) \geq \theta_{0}-C D A t .
$$

Thus $\theta(t)$ is a continuous function and for $t$ small enough $\left(t \leq 2 \theta_{0} / C A D\right.$ $\left.=: T_{2}\right)$ the condition $\theta(u)>\theta_{0} / 2$ is satisfied, which allows us to apply a fixed point theorem.

The conditions above guarantee that the operator $\mathcal{T}$ is continuous. Hence the nonlinear operator transforms $\mathcal{W}$ into itself, and is continuous. The existence of a fixed point of $\mathcal{T}$, which solves the problem (13)-(15), is a consequence of the Schauder theorem applied to the compact and convex subset $\mathcal{W} \subset \mathcal{X}$. The positivity and uniqueness of $u$ can be proved as in [6].

2.2. Global existence of solutions. Before we prove the existence of solutions for any $t>0$, we will recall some estimates for the temperature, $|u \log u|_{1}$ and $|\nabla \varphi|_{2}$. We have

$$
0<\theta_{1}<\theta<\theta_{2}<\infty,
$$

const $=0+q_{0} \log \theta_{1} \leq \int_{\Omega} u \log u=W(t)+q_{0} \log \theta \leq W(0)+q_{0} \log \theta_{2}=$ const, and

$$
0<\int_{\Omega}|\nabla \varphi|^{2}=\int_{\Omega} u \varphi=2 E-2 q_{0} \theta<2 E-2 q_{0} \theta_{2} .
$$

A useful lemma from [4] will be the main tool in the proof of global solvability.

Lemma 2.4 ([4, Th. 3]). For any $v \in H^{1}(\Omega), \Omega \subset \mathbb{R}^{2}$ and each $\varepsilon>0$, there exists a constant $C_{\varepsilon}$ such that

$$
|v|_{3}^{3} \leq \varepsilon\|v\|_{2}^{2}|v \log | v||_{1}+C_{\varepsilon}|v|_{1} .
$$


THEOREM 2.5. For every $\left\langle u_{0}, \theta_{0}\right\rangle$ with finite energy (3) and finite entropy (11), local solutions of the problem (13)-(15) in two-dimensional bounded domains can be continued for all $t \geq 0$.

Proof. Using a priori estimates for $|u \log u|_{1}$ and $|\nabla \varphi|_{2}$ due to the energy inequality we have

$$
\begin{aligned}
\frac{1}{2} \frac{d}{d t}|u|_{2}^{2}+|\nabla u|_{2}^{2} & \leq C|\nabla u|_{2}|u|_{3}|\nabla \varphi|_{6} \leq C|\nabla u|_{2}|u|_{3}^{3 / 2}|\nabla \varphi|_{2}^{1 / 2} \\
& \leq C\|u\|_{1}\left(\varepsilon\|u\|_{1}^{2}|u \log u|_{1}+C_{\varepsilon}|u|_{1}\right)^{1 / 2}|\nabla \varphi|_{2}^{1 / 2} \\
& \leq C\|u\|_{1}^{2} \varepsilon+C\|u\|_{1}|u|_{1}^{1 / 2} \leq C\|u\|_{1}^{2} \varepsilon+\frac{1}{4}\|u\|_{1}^{2}+C|u|_{1} \\
& \leq \frac{1}{2}\|u\|_{1}^{2}+C|u|_{1}
\end{aligned}
$$

for some constant $C=C\left(\theta_{0}\right)$.

Adding $|u|_{2}^{2}-\frac{1}{2}|\nabla u|_{2}^{2}$ to both sides we get

$$
\begin{aligned}
\frac{d}{d t}|u|_{2}^{2}+2|u|_{2}^{2}+|\nabla u|_{2}^{2} & \leq 3|u|_{2}^{2}+C q_{0} \leq C\|u\|_{1}|u|_{1}+C q_{0} \\
& \leq \frac{1}{2}\|u\|_{1}^{2}+C q_{0}\left(q_{0}+1\right) \leq\|u\|_{1}^{2}+C q_{0}\left(q_{0}+1\right) .
\end{aligned}
$$

Let us notice that the second inequality is valid only for $n=2$. Thus we arrive at

$$
\frac{d}{d t}|u|_{2}^{2}+|u|_{2}^{2} \leq C
$$

which yields the uniform boundedness of the norm $|u(t)|_{2}$.

So the solution obtained in the previous section can be continued for all $t \geq 0$.

To prove global solvability in the three-dimensional case we cannot use Lemma 2.4 (valid only for $n=2$ ), and estimates using the Sobolev-Gagliardo-Nirenberg inequalities do not work for the term on the right hand side of the energy inequality. In the analogous case in the isothermal model (see [1]) the only way to prove existence of global solutions was to connect them with a stationary solution. Unfortunately, in this model this idea cannot be used since we are not able to estimate $|u / \theta-U / \Theta|$ by const $|u-U|$.

\section{Stationary solutions}

Radially symmetric solutions. Considering stationary solutions we assume that $\Omega=\{|x| \leq R\}$ is a ball of radius $R$, and we look for radially symmetric solutions of (4)-(7), (9), (10). In this case the problem reads 


$$
\begin{aligned}
& u_{t}=r^{1-n}\left(r^{n-1}\left(u_{r}+\frac{u}{\theta}(\varphi+V)_{r}\right)\right)_{r}, \\
& r^{1-n}\left(r^{n-1} \varphi_{r}\right)_{r}=-u, \\
& \varphi_{r}(0, t)=0, \quad u_{r}(0, t)=0, \\
& \varphi(R, t)=0, \quad u_{r}(R, t)+\frac{u(R, t)}{\theta(t)} \varphi_{r}(R, t)=0, \\
& u(r, 0)=u_{0}(r) .
\end{aligned}
$$

Let $\widetilde{Q}(r, t)$ denote the total charge of moving ions in the ball of radius $r$ at time $t$, i.e.

$$
\widetilde{Q}(r, t)=\sigma_{n} \int_{0}^{r} s^{n-1} u(s, t) d s
$$

It is easy to check that $Q=q^{*}+\widetilde{Q}$ satisfies the equation

$$
Q_{t}=Q_{r r}-(n-1) r^{-1} Q_{r}-\left(\sigma_{n} \theta\right)^{-1} r^{1-n} Q Q_{r}
$$

The last equation has the following scaling property: if $Q$ is a solution then $Q(r, t)=\lambda^{2-n} Q\left(\lambda r, \lambda^{2} t\right)$ is also a solution. Hence, without loss of generality, we can consider our problem on the interval $[0,1]$ with the boundary and initial data

$$
\begin{aligned}
& Q(0, t)=q^{*}, \\
& Q(1, t)=q^{*}+q_{0}=: \bar{q}, \\
& Q(r, 0)=Q_{0}(r),
\end{aligned}
$$

where $Q_{0}(r)$ is a given, nonnegative, nondecreasing function on $[0,1]$ such that $Q_{0}(0)=q^{*}, Q_{0}(1)=\bar{q}$.

The physical motivation to study radially symmetric solutions is as follows.

For $n=2$ the system (19)-(22) describes the situation when the moving ions are confined to the infinite cylinder $\left\{(x, y, z): x^{2}+y^{2} \leq 1\right\}$ with fixed charge density $q^{*}$ on the $z$-axis.

The case $n=3$ corresponds to the physical model of an electrolyte contained in the unit ball in $\mathbb{R}^{3}$ with a fixed point charge $q^{*}$ at the origin.

Stationary radially symmetric solutions. If $Q(r)$ denotes a stationary radially symmetric solution to the problem (19)-(22) in $\Omega=B_{1}(0)$, then $Q(r)$ satisfies

$$
\begin{gathered}
Q_{r r}(r)-(n-1) r^{-1} Q_{r}(r)-(\Theta \sigma)_{n}^{-1} r^{1-n} Q(r) Q_{r}(r)=0 \\
Q(0)=q^{*}, \quad Q(1)=q_{0}+q^{*}=: \bar{q}
\end{gathered}
$$


with the total charge $q_{0}$ and the total energy $E$ given by

$$
E=q_{0}(\Theta+V(1))+\frac{1}{2} \sigma_{n}^{-1} \int_{0}^{1} r^{1-n}\left(Q(r)-q^{*}\right)\left(Q(r)+q^{*}\right) d r .
$$

The equation (23) (as mentioned earlier) has the following scaling property: if $Q$ is a solution then so is $\widetilde{Q}(r)=R^{2-n} Q(R r)$.

Hence if $\widetilde{Q}$ and $Q$ are solutions on $[0,1]$ and $[0, R]$ respectively, then they satisfy the following boundary conditions:

$$
\begin{array}{ll}
\widetilde{Q}(0)=q^{*}, & \widetilde{Q}(1)=\bar{q}, \\
Q(0)=R^{n-2} q^{*}, & Q(R)=R^{n-2} \bar{q} .
\end{array}
$$

Notice that in the two-dimensional case both $Q$ and $\widetilde{Q}$ satisfy the same boundary conditions

$$
\widetilde{Q}(0)=q^{*}=Q(0), \quad \widetilde{Q}(1)=\bar{q}=Q(R) .
$$

For fixed energy $E$ we have

$$
\begin{aligned}
E & =q_{0} \Theta+\frac{1}{2} \int_{0}^{R} Q^{2}(r) r^{1-n} \sigma_{n}^{-1} d r+q_{0} V(R)+q^{*} \sigma_{n}^{-1} \int_{0}^{R} r^{1-n} Q(r) d r \\
& =q_{0} \Theta+\frac{1}{2} R^{n-2} \int_{0}^{1}(\widetilde{Q}(\varrho))^{2} \sigma_{n}^{-1} d \varrho+q_{0} V(R)+q^{*} \sigma_{n}^{-1} \int_{0}^{1} \varrho^{1-n} \widetilde{Q}(\varrho) d \varrho,
\end{aligned}
$$

thus denoting by $E_{R}$ and $E_{1}$ the energy of the solution $Q$ and $\widetilde{Q}$, respectively, for $n=2$ (for the same temperature $\theta$ treated as a parameter) we have

$$
E_{R}=E_{1}-q_{0} q^{*} \frac{1}{2 \pi} \log R
$$

This means that in the two-dimensional case it is enough to find solutions on $[0,1]$. Such a solution (after rescaling) gives a solution on $[0, R]$ with the same temperature $\theta$ but with energy $E$ equal $E_{1}-q_{0} q^{*} \frac{1}{2 \pi} \log R$. Hence, without loss of generality, we will consider our problem on the interval $[0,1]$ with the boundary data (24) and energy (25).

In the three-dimensional case the situation is a bit more complicated. Since we do not know an explicit formula for the solution $Q$ we cannot get an explicit formula for $E(\theta)$. So we must act differently (we will consider this case in the next section).

For $n=2$ the stationary solutions to (19)-(22), (25) can be expressed in analytic form. In dimension 3 such a form of stationary solutions is not known and probably the equation is not integrable. In this case we resort to the phase plane method to prove the existence and uniqueness of solutions. The same method, though unnecessary, can also be used in the much simpler two-dimensional case. Moreover, the phase plane method allows us to find 
a very simple proof of existence and uniqueness when analytic arguments require some tedious calculations.

3.1. Two-dimensional case. For $n=2$ stationary solutions to the problem (19)-(22) in $\Omega=B_{1}(0)$ satisfy

$$
Q_{r r}-r^{-1} Q_{r}-(2 \pi \Theta)^{-1} r^{-1} Q Q_{r}=0
$$

with the boundary conditions

$$
Q(0)=q^{*}, \quad Q(1)=\bar{q}=: q^{*}+q_{0},
$$

and the energy given by

$$
E=q_{0} \Theta+\frac{1}{4 \pi} \int_{0}^{1}\left(Q(r)-q^{*}\right)\left(Q(r)+q^{*}\right) \frac{1}{r} d r .
$$

The idea of the proof is as follows. First we choose $q_{0}$ and $q^{*}$, and solve (26)-(27) treating $\theta$ as a parameter. Then we show that the energy $E$ depending on the (unique) solution $Q=Q(\theta)$ is a strictly increasing function of the temperature. So, given $E$ we find a unique $\theta=\theta\left(E, q_{0}, q^{*}\right)$, and finally we get a solution $Q=Q(\theta)=Q\left(E, q_{0}, q^{*}\right)$. Monotonicity of $E(\theta)$ guarantees the uniqueness of $Q$.

The equal signs case $\left(q^{*}>0\right)$

THEOREM 3.1. For any $q^{*}, q_{0}>0$ and energy $E>0$ there exists a unique solution $\langle Q(r), \theta\rangle$ to the problem (26)-(28).

Proof. Using the existence of the first integral $2 r Q_{r}-4 Q-\frac{1}{2 \pi \Theta} Q^{2}=C$ of the equation (26) we obtain the solution in the form

$$
Q(r)=-\left(8 \pi \Theta+q^{*}\right)+\frac{8 \pi \Theta+2 q^{*}}{1-\frac{q_{0}}{\bar{q}+q^{*}+8 \pi \Theta} r^{2+q^{*} / 2 \pi \Theta}} .
$$

The energy for such a solution is given by the formula

$$
E=2 q_{0} \Theta+8 \pi \Theta^{2} \log \left(1-\frac{q_{0}}{8 \pi \Theta+q^{*}+\bar{q}}\right) .
$$

Observe that the energy $E$ is a strictly increasing function of $\Theta$. Indeed,

$$
\frac{\partial E}{\partial \Theta}>0, \quad \lim _{\Theta \rightarrow \infty} E(\Theta)=\infty, \quad \lim _{\Theta \rightarrow 0} E(\Theta)=0 .
$$

These conditions imply that for all values of $q^{*}, q_{0}>0$ and energy $E>0$ there exists a unique solution of (26)-(28).

REMARK 3.2. According to the considerations of the previous section, there exists a unique solution $Q(r), \theta$ to the problem $(26),(28)$ on $[0, R]$ for any $q^{*}, q_{0}>0$ and energy $E>E_{0}=-\frac{q^{*} q_{0}}{2 \pi} \log R$. 
The opposite signs case $\left(q^{*}<0\right)$. To simplify notation we change $q^{*}<0$ to $-q^{*}$ and assume that $q^{*}>0$. In this case we solve the following problem:

$$
Q_{r r}-r^{-1} Q_{r}-(2 \pi \Theta)^{-1} r^{-1} Q Q_{r}=0
$$

with the boundary conditions

$$
Q(0)=-q^{*}, \quad Q(1)=\bar{q}=q_{0}-q^{*},
$$

and the given energy

$$
E=q_{0} \Theta+\frac{1}{4 \pi} \int_{0}^{1}\left(Q(r)-q^{*}\right)\left(Q(r)+q^{*}\right) \frac{1}{r} d r .
$$

THEOREM 3.3. For all values of $q_{0}>0, q^{*}>0$ and energy $E \in(-\infty, \infty)$ there exists a unique solution $\langle Q(r), \theta\rangle$ of the problem (29)-(31).

Proof. Just as in the previous case, we can get an analytical solution. Integrating the equation (29) we get

$$
2 r Q_{r}-4 Q-\frac{1}{2 \pi \Theta} Q^{2}=C .
$$

Now we consider two cases: $q^{*}<4 \pi \Theta$ and $q^{*}=4 \pi \Theta$ (for $q^{*}>4 \pi \Theta$ the above equation is not integrable due to the boundary conditions (30)).

For $q^{*}<4 \pi \Theta$ the solution of $(29)-(30)$ has the form

$$
Q(r)=-\left(8 \pi \Theta-q^{*}\right)+\frac{8 \pi \Theta-2 q^{*}}{1-\frac{q_{0}}{\bar{q}-q^{*}+8 \pi \Theta} r^{2-q^{*} / 2 \pi \Theta}} .
$$

For $q^{*}=4 \pi \Theta$ we have

$$
Q(r)=-4 \pi \Theta\left(1-\frac{q_{0}}{4 \pi \Theta-q_{0} \log r}\right)=-q^{*}\left(1-\frac{q_{0}}{q^{*}-q_{0} \log r}\right) .
$$

The energy $E$ for $q^{*}<4 \pi \Theta$ is given by the formula

$$
\begin{aligned}
E & =2 q_{0} \Theta+8 \pi \Theta^{2} \log \left(1-\frac{q_{0}}{8 \pi \Theta-q^{*}+\bar{q}}\right) \\
& =2 q_{0} \Theta-8 \pi \Theta^{2} \log \left(1+\frac{q_{0}}{8 \pi \Theta-2 q^{*}}\right),
\end{aligned}
$$

while for $q^{*}=4 \pi \Theta$ the energy is equal to $-\infty$.

Now let us examine the energy function $E$. Since

$$
\lim _{\Theta \rightarrow \infty} \Theta \log \left(1-\frac{q_{0}}{8 \pi \Theta-q^{*}+\bar{q}}\right)=-\frac{q_{0}}{8 \pi},
$$

we have

$$
\lim _{\Theta \rightarrow \infty} E(\Theta)=\infty
$$


Now we should examine $\lim _{\Theta \rightarrow q^{*} / 4 \pi} E(\Theta)$ (since the condition $q^{*} / 4 \pi<\Theta$ must be satisfied). We have

$$
\lim _{\Theta \rightarrow q^{*} / 4 \pi} E(\Theta)=-\infty
$$

So $E:(-\infty, \infty) \rightarrow(-\infty, \infty)$.

To prove the uniqueness of the solution it is enough to check the monotonicity of $E(\Theta)$ which is obvious since $\partial E / \partial \Theta>0$.

3.2. Three-dimensional case. For $n=3$ a stationary solution $Q$ of the problem (19)-(22) satisfies

$$
\begin{gathered}
Q_{r r}-2 r^{-1} Q_{r}-\Theta^{-1}(4 \pi)^{-2} r^{-2} Q Q_{r}=0, \\
Q(0)=q^{*} \\
Q(1)=\bar{q}
\end{gathered}
$$

and the energy is given by

$$
E=q_{0}\left(\Theta+q^{*}(4 \pi)^{-1}\right)+\frac{1}{2}(4 \pi)^{-1} \int_{0}^{1}\left(Q(r)-q^{*}\right)\left(Q(r)+q^{*}\right) r^{-2} d r
$$

The case of equal signs $\left(q^{*}>0\right)$. Since the analytical solution of the problem (32)-(35) is not known we use the phase plane method.

ThEOREM 3.4. For all $q^{*}, q_{0}>0$ and energy $E>E_{0} \geq 0$ there exists a solution of (32)-(35).

Proof. Introducing the new variables

$$
v(s)=(4 \pi \Theta)^{-1} Q_{r}(r), \quad w(s)=(4 \pi \Theta)^{-1} Q(r) / r, \quad s=\log r,
$$

we transform the problem of existence of stationary solutions to (32)-(34) into the problem

$$
\begin{gathered}
w^{\prime}=v-w, \quad '=\frac{d}{d s}, \\
v^{\prime}=v(2+w), \\
w(0)=(4 \pi \Theta)^{-1} \bar{q}, \quad \lim _{s \rightarrow-\infty} w(s) e^{s}=(4 \pi \Theta)^{-1} q^{*} .
\end{gathered}
$$

Figure 1 shows the phase portrait of (37)-(38).

The origin is a saddle (with two eigenvalues 2 and -1 ), $\gamma$ is its unstable manifold. Let us choose any $\bar{q}$. Let $(w(s), v(s))$ be a solution to (37)-(38) with initial data $w(0)=(4 \pi \Theta)^{-1} \bar{q}, v(0)=v \in[0, \bar{v})$ (see Fig. 1).

This solution is defined on some maximal interval $(\alpha, \beta)$. It follows from (37) that $\alpha=-\infty$. The function $e^{s} w(s)$ is increasing (since its derivative is $e^{s} v(s)$ ), hence $\lim _{s \rightarrow-\infty} e^{s} w(s)=: L(v)$ exists. Obviously $L(0)=\bar{q}$ and $L(\bar{v})=0$, so there exists $v^{*} \in[0, \bar{v})$ such that $L\left(v^{*}\right)=q^{*}$. The uniqueness of $v^{*}$ follows from the monotonicity of $L(v)$. 


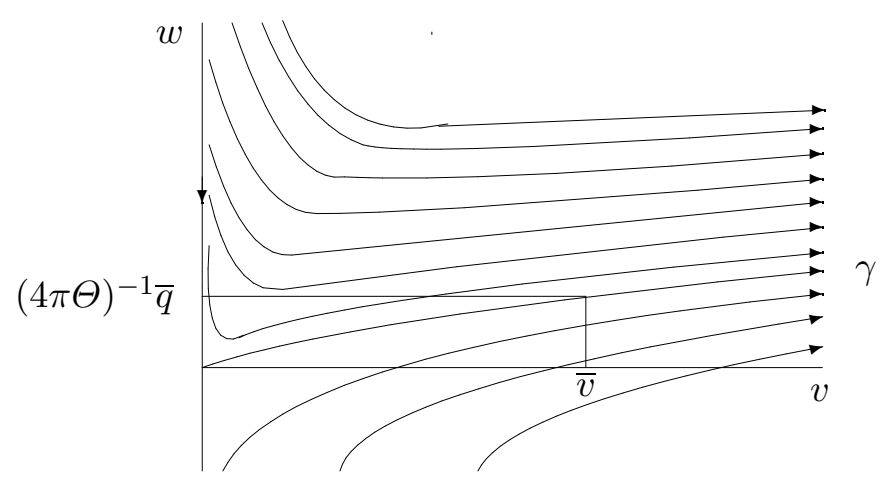

Fig. 1. The phase portrait of (37)-(38)

The solution obtained is well defined since the integral in the formula for the energy $E$ exists. Indeed, dividing (32) by $Q_{r}$ we get

$$
\begin{aligned}
\frac{Q_{r r}}{Q_{r}}-2 r^{-1}-\Theta^{-1}(4 \pi)^{-2} r^{-2} \bar{q} \leq 0 & =\frac{Q_{r r}}{Q_{r}}-2 r^{-1}-\Theta^{-1}(4 \pi)^{-2} r^{-2} Q \\
& \leq \frac{Q_{r r}}{Q_{r}}-2 r^{-1}-\Theta^{-1}(4 \pi)^{-2} r^{-2} q^{*}
\end{aligned}
$$

Integrating over $[\varepsilon, r]$ we arrive at

$$
\frac{Q_{r}(r)}{r^{2}} \exp \left(-\frac{\bar{q}}{\Theta(4 \pi)^{2}}\left(\frac{1}{\varepsilon}-\frac{1}{r}\right)\right) \leq \frac{Q_{r}(\varepsilon)}{\varepsilon^{2}} \leq \frac{Q_{r}(r)}{r^{2}} \exp \left(-\frac{q^{*}}{\Theta(4 \pi)^{2}}\left(\frac{1}{\varepsilon}-\frac{1}{r}\right)\right) .
$$

The second inequality implies that $\lim _{\varepsilon \rightarrow 0} Q_{r}(\varepsilon) / \varepsilon^{2}=0$, so the integral $\int_{0}^{1}\left(Q-q^{*}\right)\left(Q+q^{*}\right) r^{-2}$ exists.

Moreover the same inequality implies that $Q_{r}(\varepsilon) / \varepsilon^{2} \rightarrow 0$ as $\Theta \rightarrow 0$, so $E(\Theta) \rightarrow E_{0} \geq 0$ as $\Theta \rightarrow 0$.

For $\Theta \rightarrow \infty$ we have $E \rightarrow \infty$ so $E:(0, \infty) \rightarrow\left(E_{0}, \infty\right)$.

REMARK 3.5. The idea of the proof for $n=3$ is also valid for $n>3$. Indeed, introducing new variables:

$$
\begin{aligned}
v(s) & =\left(\sigma_{n} \Theta\right)^{-1} r^{3-n} Q_{r}(r), \\
w(s) & =\left(\sigma_{n} \Theta\right)^{-1} r^{2-n} Q(r),
\end{aligned}
$$

where $s=\log r$, we get the following problem:

$$
\begin{gathered}
w^{\prime}=(2-n) w+v, \\
v^{\prime}=v(2+w), \\
w(0)=\left(\sigma_{n} \Theta\right)^{-1} \bar{q}, \quad \lim _{s \rightarrow-\infty} w(s) e^{(n-2) s}=\left(\sigma_{n} \Theta\right)^{-1} q^{*} .
\end{gathered}
$$

The phase portrait for (42)-(44) is the same as for $n=3$. 
The opposite signs case $\left(q^{*}<0\right)$. As in the two-dimensional case, we change $q^{*}<0$ to $-q^{*}$ and assume that $q^{*}>0$. So stationary solutions satisfy

$$
Q_{r r}-2 r^{-1} Q_{r}-\Theta^{-1}(4 \pi)^{-2} r^{-2} Q Q_{r}=0
$$

with the boundary conditions

$$
\begin{aligned}
& Q(0)=-q^{*}, \\
& Q(1)=q_{0}-q^{*},
\end{aligned}
$$

and the energy given by

$$
E=q_{0}\left(\Theta-q^{*}(4 \pi)^{-1}\right)+\frac{1}{2}(4 \pi)^{-1} \int_{0}^{1}\left(Q(r)-q^{*}\right)\left(Q(r)+q^{*}\right) r^{-2} d r .
$$

THEOREM 3.6. For any values of $q^{*}, q_{0}>0$ there exists no solution to the problem (45)-(48).

Proof. Let us introduce the new variable $y=r^{3}$. Taking $Q=Q+q^{*}$ we transform (45) into

$$
3 y^{4 / 3} Q_{y y}-(4 \pi \Theta)^{-1}\left(Q-q^{*}\right) \frac{Q_{y}}{2}=0 .
$$

Suppose that there exists a solution $Q \not \equiv 0$ of (49). Since $Q(0)=0$ we see for small $y\left(y<y_{1}\right)$ that $Q(y) \leq q^{*} / 2 \rightarrow Q-q^{*} \leq-q^{*} / 2$. So

$$
0 \geq 3 y^{4 / 3} Q_{y y}-(4 \pi \Theta)^{-1} \frac{q^{*}}{2} \frac{Q_{y}}{2} .
$$

Dividing by $3 Q_{y} y^{4 / 3}$ we get

$$
\frac{d}{d y}\left(\log Q_{y}+C_{1}(\Theta) y^{-1 / 3}\right) \leq 0
$$

which implies monotonicity of the above function, so

$$
\log Q_{y}+C_{1}(\Theta) y^{-1 / 3} \geq C_{2}(\Theta)
$$

for $y \in\left(0, y_{2}\right), y_{2} \leq y_{1}$. Integrating the inequality above we get

$$
Q(y)-Q(0) \geq \int_{0}^{y} \exp \left(-C_{1}(\Theta) x^{-1 / 3}+C_{2}(\Theta)\right) d x .
$$

Since the function $\exp \left(x^{-1 / 3}\right)$ is not integrable near 0 , we obtain $Q(y) \geq \infty$. The singularity of $Q$ near 0 implies that we are not able to define a solution, so there is no solution to our problem.

Acknowledgements. The author would like to thank T. Nadzieja for numerous discussions during the preparation of this paper. 


\section{References}

[1] P. Biler, Existence and asymptotics of solutions for a parabolic-elliptic system with nonlinear no-flux boundary conditions, Nonlinear Anal. 19 (1992), 1121-1136.

[2] P. Biler, J. Dolbeault, M. J. Esteban and G. Karch, Stationary solutions, intermediate asymptotics and large time behaviour of type II Streater's models, Adv. Differential Equations 6 (2001), 461-480.

[3] P. Biler, J. Dolbeault, M. J. Esteban, P. A. Markowich, T. Nadzieja, Steady states for Streater's energy-transport models of self-gravitating particles, in: Transport in Transition Regimes, N. Ben Abdallah et al. (eds.), IMA Vol. Math. Appl. 135, Springer, 2003, 37-56.

[4] P. Biler, W. Hebisch and T. Nadzieja, The Debye system: existence and long time behavior of solutions, Nonlinear Anal. 23 (1994), 1189-1209.

[5] P. Biler, A. Krzywicki and T. Nadzieja, Self-interaction of Brownian particles coupled with thermodynamic processes, Rep. Math. Phys. 42 (1998), 359-372.

[6] P. Biler and T. Nadzieja, A class of nonlocal parabolic problems occurring in statistical mechanics, Colloq. Math. 66 (1993), 131-145.

[7] -, - A singular problem in electrolytes theory, Math. Methods Appl. Sci. 20 (1997), 767-782.

[8] - - - Global and exploding solutions in a model of self-gravitating systems, Rep. Math. Phys. 52 (2003), 205-225.

[9] -, 一, Nonlocal parabolic problems in statistical mechanics, Nonlinear Anal. 30 (1997), 5343-5350.

[10] - - - Structure of steady states for Streater's energy-transport models of gravitating particles, Topol. Methods Nonlinear Anal. 19 (2002), 283-301.

[11] P.-H. Chavanis, J. Sommeria and R. Robert, Statistical mechanics of two-dimensional vortices and collisionless stellar systems, Astrophys. J. 471 (1996), 385.

[12] D. Gilbarg and N. S. Trudinger, Elliptic Partial Differential Equations of Second Order, Springer, Berlin, 2001.

[13] T. Nadzieja and A. Raczyński, A singular radially symmetric problem in electrolytes theory, Appl. Math. (Warsaw) 25 (1998), 101-112.

[14] - - - Radially symmetric solutions of the Poisson-Boltzmann equation with a given energy, ibid. 27 (2000), 465-473.

[15] I. Rubinstein, Electro-Diffusion of Ions, SIAM Stud. Appl. Math. 11, SIAM, Philadelphia, 1990.

[16] I. Rubinstein and L. Rubinstein, Partial Differential Equations in Classical Mathematical Physics, Cambridge Univ. Press, Cambridge, 1993.

[17] R. F. Streater, Dynamics of Brownian particles in a potential, J. Math. Phys. 38 (1997), 4570-4575.

[18] - , The Soret and Dufour effects in statistical dynamics, Proc. Roy. Soc. London Sect. A 456 (2000), 205-211.

Institute of Mathematics

University of Wrocław

Pl. Grunwaldzki 2/4

50-384 Wrocław, Poland

E-mail: Andrzej.Raczynski@math.uni.wroc.pl

Received on 11.4.2003;

revised version on 3.12.2004 